\title{
scripted
}

Volume 13, Issue 3, December 2016

\section{CONFERENCE REPORT: \\ LIMINAL SPACES SYMPOSIUM AT IAB 2016: WHAT DOES IT Mean to Regulate in the PUblic Interest?}

\author{
Annie Sorbie ${ }^{*}$
}

\begin{abstract}
This Conference Report summarises a Wellcome Trust-sponsored symposium held at the 13th World Congress of the International Association of Bioethics, held in Edinburgh 14-17 June 2016 (IAB2016). This symposium was curated by the Liminal Spaces Project, which is conducted under the auspices of the JK Mason Institute for Medicine, Life Sciences and the Law at the University of Edinbugh School of Law, and sought to address the question: "What does it mean to regulate in the public interest?"
\end{abstract}

DOI: $10.2966 /$ scrip.130316.374

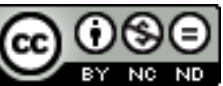

(C) Annie Sorbie 2016. This work is licensed under a Creative Commons Licence. Please click on the link to read the terms and conditions.

* PhD Candidate and Member of Liminal Spaces Project, School of Law, University of Edinburgh. 


\section{Introduction}

As reported in Volume 13:2 of SCRIPTed, the 13th World Congress of the International Association of Bioethics, held in Edinburgh in June 2016, explored the theme "Individuals, Public Interests and Public Goods." The Liminal Spaces Project, ${ }^{2}$ an interdisciplinary initiative sponsored by the Wellcome Trust and conducted under the auspices of the JK Mason Institute for Medicine, Life Sciences and the Law at the University of Edinbugh School of Law, took up this theme by posing the following question to scholars from a range of disciplines: how can health research be regulated in the public interest? This Conference Report summarises those responses and, in doing so, draws out two key themes that ran throughout the presentations.

\section{The public interest - a contested concept}

The public interest has been described as a “...vague, impalpable but all-controlling consideration... ."3 The notion of public interest took on scholarly interest in postWorld War II America, where it was both critiqued and praised. During this time, for example, the political scientist Frank Sorauf concluded that this "elusive catchword" 4 would do well to join a list of ambiguous words and phrases "which never would be missed." Against this, proponents of the public interest took a different view, making bold claims to the effect that "...the public interest is the central concept of a civilised polity."

Despite this historical divergence of views, in health research the public interest is embedded throughout regulation. For example, in the context of biomedical research involving human subjects, consideration of the relationship between individual and public health was central to the ethical guidance adopted in 1964 in the original Declaration of Helsinki, which was formulated in the wake of atrocities perpetrated by Nazi physicians. ${ }^{7}$ In the realm of data protection, the public interest forms part of the route to the lawful processing of personal information, both in accordance with the EU's Data Protection Directive 95/46/EC and the common law duty of confidentiality. A third example is the mandate of the UK's Health Research

\footnotetext{
${ }^{1}$ See ES Dove et al, "Conference Report: 13th World Congress of the International Association of Bioethics, Edinburgh 14-17 June 2016 (IAB2016)" (2016) 13 SCRIPTed 202-209.

${ }^{2}$ Liminal Spaces Project, available at http://www.liminalspaces.ed.ac.uk/ (accessed 8 Dec 16).

${ }^{3}$ G Colm, "The Public Interest: Essential Key to Public Policy" in C Friedrich (ed), Nomos V (New York: Atherton Press, 1962) 115-128, at 115.

${ }^{4}$ F Sorauf, "The Conceptual Muddle” in C Friedrich, see note 3 above, 183-190, at 187.

${ }^{5}$ Ibid, 190.

${ }^{6}$ S Bailey, "The Public Interest: Some Operational Dilemmas" in C Friedrich, see note 3 above, 96106, at 106.

7 JR Williams, "The Declaration of Helsinki and Public Health" 86 Bulletin of the World Health Organization 650-652.
} 
Authority, which is to protect and promote the interests of patients and the public in health and social care research. ${ }^{8}$

However, while the public interest may be replete in health research regulation (HRR), HRR-specific literature follows that of the political science commentary generations ago, suggesting that it remains "a notoriously uncertain idea." there is plenty of room to tease out what this concept may mean, and IAB2016 presented a timely opportunity for the Liminal Spaces Project to engage with this ubiquitous, but somewhat neglected, concept. This was a natural fit in view of the Liminal Spaces Project's commitment to providing the first-ever integrated, interdisciplinary and cross-cutting analysis of HRR which confronts "liminal" regulatory spaces and promotes an holistic approach thus far absent in HRR.

\section{The Liminal Spaces symposium: an overview}

The Wellcome Trust-sponsored LS symposium brought together five expert speakers: Leslie Stevens (University of Edinburgh); David Townend (Maastricht University); Alena Buyx (University of Kiel); Annette Rid (King's College London) and Mark Taylor (University of Sheffield). Their presentations drew on philosophy, law and the social sciences. In addressing HRR, their contributions touched on various aspects of this diverse field, including access to data for health research, research involving human participants, and organ donation. Each of the speakers recognised the challenges and opportunities presented in this context, where appeals to the public interest are preyed in aid of both more and less regulation, and where the public benefits of the fruits of health research are routinely pitted against individual interests in privacy, confidentiality and bodily integrity.

While approaching the concept of the public interest from different angles, there were two cross-cutting themes that united the speakers. First, it was agreed that the public interest need not be balanced against private individual interests. Rather than viewing these as competing values, the speakers explored how these could be accounted for in a conception of the other. In doing so, this underlined the need for legitimacy and transparency where the public interest is deployed by regulators, researchers or other actors. This dovetailed with the second theme, namely the implications of this for the operationalisation of the public interest in HRR, both in the management of data and the regulation of research and treatment involving human participants more broadly.

\section{Symposium presentations}

Leslie Stevens opened the symposium by outlining her research with Professor Graeme Laurie on their development of "A Public Interest Mandate to Support the Use, Sharing and Linkage of Administrative Data." Stevens set out the everincreasing demand for public sector data sharing and identified how a public interest mandate may be used to overcome barriers to data sharing. In doing so, she engaged directly with the thorny issue of operationalising the public interest in a real world context.

\footnotetext{
${ }^{8}$ The HRA was first established in December 2011, and then as a statutory Non-Departmental Public Body as of 1 January 2015.

${ }^{9} \mathrm{M}$ Taylor, Genetic Data and the Law: A Critical Perspective on Privacy Protection (Cambridge, CUP, 2012) at 29.
} 
Stevens drew on her and Laurie's previous work with data initiatives and research communities, and presented three key points that had emerged: (1) there exists a "culture of caution" around data sharing; (2) organisational self-interest surrounds public sector data; and (3), perhaps most surprisingly, the key barriers to data sharing are not legal, but cultural.

Stevens expanded upon this final point to elucidate how law can be used as a shield for other concerns. For example, she and Laurie found that legal complexity may be conflated with impermissibility. A binary approach of "consent or anonymise" was also in evidence. In other words, options beyond either asking individuals or anonymising data before sharing were not considered by data controllers, even though other routes to lawful processing may be available. Finally, in the interpretation of legislation (for example, the Data Protection Act 1998), Stevens highlighted an unwarranted emphasis on how this operates to protect the use of personal data, with insufficient attention being paid to how this legislation can also facilitate the use of personal data.

In probing these barriers to data sharing, Stevens distinguished between real and perceived risks and set out a decision-making matrix for data controllers. In looking to achieve culture change, Stevens proposed a role for a public interest mandate for public sector data management. This multi-faceted approach was underpinned by: (i) scrutiny of data sharing readiness; (ii) an overt commitment to considering the public interest in any given context; (iii) meaningful public engagement with an emphasis on whether a social licence to share is in existence; (iv) implementation of a proportionate approach, including in the evaluation of the risks and benefits of any given course of action; and ( $\mathrm{v}$ ) managing uncertainty by keeping data sharing arrangements - and the role of the public interest in those arrangements - under continuous review. Thus, Stevens provided a path in which problematic organisational cultures can be tackled and a paradigm shift achieved in how public authorities value and act upon data.

Professor David Townend presented on "Personal Responsibility and the Public Interest," proving a rich account of the interplay between personal and public interests. Having first identified the potential benefits of the public interest including its ability to leave room for discretion, flexibility, and the integration of community rights - Townend also touched on the some of the issues that had also previously vexed Frank Sorauf. These included problems of definition, locating the source of the authority to decide what is, and indeed is not, in the public interest, and the sense of "otherness" that this can engender.

In constructing the public interest, Townend looked to a refined utilitarian calculus. Here he went beyond an approach where the (arguably limited) harms to an individual are balanced against the (potentially overwhelming) benefits to the public, and instead framed this on a 1:1 basis, as between the actual individual and the foreseeable "other." As Townend has highlighted in previous work, this does not go towards resolving whose rights should prevail but places matters on a "...fair and equitable footing. $" 10$

\footnotetext{
${ }^{10}$ D Townend, “Overriding Data Subjects' Rights in the Public Interest" in D Beyleveld et al (eds) The Data Protection Directive and Medical Research Across Europe (Hants: Ashgate, 2004) 89-101, at 100.
} 
In looking to tease out a sense of the relationship between personal and public interests, Townend traced this through the post-English Civil War philosophy of the Third Earl of Shaftesbury, referring to the concept of politeness as including a personal responsibility for self-reflection and other-centeredness. In grounding social liberalism with an undergirding of morality, Townend argued that responsibility is, in fact, integral to autonomy, a connection that only "slipped its moorings" in the monetarism and lower taxation of the 1970 and 1980s that marked a shift from liberalism to individualism.

In considering the impact of this approach on HRR, Townend turned to the question of whether an individual can have a free choice to require treatments that depend on participation in research, but refuse to participate him- or herself, even though this will not cause any significant harm to that individual. He concluded that this could only be the case under unfettered individualism, and not under iterations of (even extreme) liberalism. This he linked to a normative competence to require "active citizenship," suggesting that bioethics does not have to accept passive citizenship. Having made this point, Townend recognised that this is a two-way street and noted the reciprocal responsibilities this placed on other regulatory actors, for example, in the context of benefit sharing with research participants.

Dr Annette Rid presented on the topic "In Pursuit of Proportionality, Regulating Health Research in the Public Interest." The twin bases of Rid's argument were, first, that the ethical oversight of health research should rest on a pluralistic conception of public interest; and second, that a proportionate system of ethical oversight is the most defensible way of implementing this conception, though this is not free of challenges.

In her presentation, Rid identified the common dualistic framing of HRR as a balance between, on the one hand, the public interest in promoting valuable research, and, on the other, the individual interests and rights of research participants. In doing so, she levelled two criticisms at this standard view: first, that this framing is artificially antagonistic; and, second, that it is too narrow.

Expanding on her critique, Rid noted that the relationship between public and individual interests in health research is not solely - and perhaps not even primarily one of conflict. She highlighted how individual and public interests in health research are more intertwined than the standard view suggests. For example, individuals have an interest in systems that address our potential future health needs. Further, she noted that research is a collective enterprise that requires broad societal involvement, and that this cooperation requires public trust and the respect of individual rights.

Second, Rid suggested that this standard view assumes a very narrow conception of public interest that is confined to the value of the knowledge used to promote health. Rid pointed to other, wider public interests in health research, such as fairness in the distribution of research risks and benefits, and the public interest in wider deliberation about research priorities, particularly given public financial support of this endeavour. She concluded that this indicates a need to integrate the plurality of public interests in health research (which includes individual rights and interests).

In operationalising this approach, Rid looked to proportionate ethical oversight as the most appropriate tool. She highlighted that oversight systems should be proportionate to: (i) the importance of the public interests at stake in the given research; (ii) the extent to which these interests require protection; and also (iii) the extent to which a given form of oversight provides effective protection. Rid justified this approach on 
the grounds of fairness (i.e. treating like cases alike and avoiding duplication), utility (in that this avoids unnecessarily delaying or deterring valuable research), and also efficiency and cost. This was tempered with a healthy dose of pragmatism, however, as Rid admitted the significant challenge this could pose, given the plurality of public interests in play.

The penultimate presentation was delivered by Professor Alena Buyx and focussed on "Public Interest and Solidarity." Here Buyx drew on her joint work with Barbara Prainsack and outlined how the concept of solidarity, which has been steadily gaining currency in HRR, interrelates with the public interest.

Buyx first turned to the central challenge of regulating in the public interest, in that the key regulatory institutions and instruments in HRR focus on the individual. While this generates both conceptual and practical issues, Buyx suggested ways that solidarity can help in this regard.

Buyx traced the development of the concept of solidarity and outlined the working definition that she and Prainsack have developed. ${ }^{11}$ This is that solidarity signifies practices reflecting a commitment to carry "costs" (financial, social, emotional, or otherwise) to assist others with whom we recognise similarity in a relevant respect. In their work, Buyx set out how this has been conceptualised as operating at three levels: first at the interpersonal level (tier 1), then as group practices (tier 2), and finally at a contractual level (tier 3).

Buyx outlined how solidarity works in practice by drawing on the example of organ donation and considering the extent to which each tier of solidarity was engaged. In doing so, she drew out the implications of using solidarity as a regulatory principle to frame organ donation regulation, in that this brings to the fore the collective aspects of organ donation (i.e. what people share in common). Going further, this raised the potential for solidarity to operate as a "corrective" of regulatory individualism. Buyx noted that this was easiest to do first in the context of "soft regulation," where existing legal frameworks may be rebalanced to include solidarity prominently, alongside the protection of individual rights and interests.

From this analysis, Buyx set out how solidarity and public interest perspectives can overlap, although this is not always the case. Taking organ donation as an example, Buyx outlined how a switch to "opt-out" state regulation, as a solidaristic practice where collective implications are foregrounded, would lead to the availability of more organs, with no "loss" of willing donors. This result would also be in the public interest. However, in the example of a priority donation program (for example, in Israel a points reward system operates to motivate individuals to donate organs) this could also possibly lead to more donors, which may be in the public interest, but would not be a solidaristic practice.

Buyx concluded that not all solidaristic practices will be in the public interest, and not all public interest initiatives will be solidaristic practices. However, solidarity does take us some way towards regulation in the public interest and can provide a conceptual and practical starting point to translate the notion of public interest into policy and governance

\footnotetext{
11 B Prainsack and A Buyx, Solidarity: Reflections on an Emerging Concept in Bioethics (2011) Nuffield Council on Bioethics. See also B Prainsack and A Buyx, Solidarity in Biomedicine and Beyond (Cambridge: CUP, 2017).
} 
Dr Mark Taylor gave the final presentation, entitled "Expect, Respect, and Accept: A Triple Test for the Proper Protection of Privacy and Public Interest in the Use of Patient Confidential Data for Public Policy Objectives?" Taylor addressed some of the competing conceptions of the public interest, again highlighting the term's lack of precision that had so frustrated Sorauf and been touched on by Townend. He also spoke to the benefits and drawbacks of various theories of the public interest and helpfully summarised each. These conceptions of the public interest were as: (i) an aggregate of individual interests; (ii) a common interest (where there is a collective or universal public benefit, though not one that is necessarily uniformly applied); or (iii) a value-based concept linked to overriding interests in society (though Taylor noted the challenge inherent in locating such values in a pluralistic society).

Through interrogating these approaches, Taylor proposed that to regulate in the public interest means "to protect and trade off common interests in a way that the (affected) public has cause to accept." He drew out the common value implicated here - namely respect for individuals as free and equal persons - and the common interests in play such as the enjoyment of private and family life and good health. Further, Taylor highlighted the role of public reason, arguing that it should be possible to justify rules to those who are subject to them with reference to arguments they have reason to accept.

Taylor developed his conceptual approach by outlining how this could be operationalised by his proposed triple test: "Expect, Accept and Respect". He suggested that respect for both privacy and the public interest may be characterised by persons being provided (1) reasons to expect the use of data and (2) reasons to accept that use; and (3) that the data uses respect patient preferences. Taylor argued that legitimate invocations of both privacy and the public interest protect and constrain one another. As a result, the public interest is not something to be balanced against privacy, nor the reverse. Each can place limits upon the other because each should be accounted for in a conception of the other. Recognising the relationship between them is not to recognise the need for compromise; rather, it is to recognise the need for legitimacy.

\section{Conclusion}

The Liminal Spaces Project, with the support of the Wellcome Trust, was delighted to bring together expert speakers who contributed substantially to the core theme of IAB2016, bringing to the fore the interplay between individuals, public interests and public goods in bioethics.

Each of the symposium speakers made rich and nuanced contributions that highlighted two pervasive themes. This first is that the public interest need not be balanced against private or personal interests. Using the anthropological concept of liminality to probe this further, we would suggest that if the health research regulatory landscape is reimagined as a coherent whole, with multiple interests in play, we can continue to deepen our understand of the public interest as a concept. This leads us to the second theme that emerged, namely a commitment on the part of the speakers to reintegrate the concept of the public interest to real world practice, and to consider how this concept could deliver tangible benefits, whether this be in the context of data sharing, health research participation, proportionate governance or organ donation.

To conclude, we would also set out our statement of intent for future work on the Liminal Spaces Project. The concept of the public interest is embedded in HRR both 
in policy and in legislation. We therefore find that this previously neglected phrase demands careful examination and explanation. The range of views expressed, both by those presenting at the symposium, and more widely across IAB2016's plenary and parallel sessions, indicate to us that the concept would benefit from further intellectual scrutiny.

\section{Acknowledgements}

The Liminal Spaces Project would like to thank each of the speakers at this symposium, both for their thoughtful contributions and for their enthusiasm for and engagement with this topic. This research was supported by a Wellcome Trust Senior Investigator Award No. WT103360MA entitled "Confronting the Liminal Spaces of Health Research Regulation.” 